\title{
Hybrid Teaching vs. Traditional Teaching in Computer Engineering Courses: What works and What does not work?
}

\author{
Andy S. Peng, Robert Nelson, Cheng Liu \\ Engineering and Technology Department \\ University of Wisconsin - Stout \\ Menomonie, WI \\ \{penga, nelsonro, liuc\}@uwstout.edu
}

\author{
Jia-Ling Lin \\ STEM Education Center \\ University of Minnesota \\ Minneapolis, MN \\ jllin@umn.edu
}

\author{
Lynn M. Meredith \\ Thomson Reuters \\ Eagan, MN \\ lmmeredith@comcast.net
}

\begin{abstract}
This study compares student learning outcomes from two instructional approaches: hybrid vs. traditional teaching method. The study applies a previously developed framework in order to assess the learned curriculum for the same upper division computer engineering course. It also analyzes how it is aligned with the intended curriculum. The same undergraduate computer engineering course was taught by two different instructors during two different semesters. Both instructors have extensive technical background and many years of practical engineering experiences in the related field. Both classes used an identical textbook, delivered similar set of course topics, had similar lab setup, required homework assignments as well as a semester-long team project. The key difference is the use of online lectures. Based on students' responses to series of surveys and the result of final grades, this study compares their development in content knowledge and cognitive abilities to determine the effectiveness of the instructional approach. The study provides an interest in finding ways to truly utilize technology for improving student learning, particularly their development of cognitive abilities. The study also seeks the impact of the technology on lecturing styles and in-classroom dynamics. Furthermore, this study will help gain insights into instructional approaches concerning teaching and learning along the technological dimension.
\end{abstract}

\section{Introduction}

In order to effectively deliver course content covering a broad range of topics and facilitate interactive learning activities, engineering courses have been traditionally delivered in classroom settings until recent years when Internet technologies have become an integral part of the higher education system $^{[1]}$. Internet technologies play a significant role in the recent development of engineering education system. By leveraging the benefits of online training and traditional inclass training, hybrid instructional approaches have gained increasing support for various reasons. Proponents seize the opportunity to effectively integrate live classroom activities with online learning and instructions in order to maximize teaching and learning potential. The setup enables students to learn course content while allowing the instructors to efficiently control over time, place, and pace. Several challenges remain in such course settings. In previous studies ${ }^{[2][3]}$, we've developed a framework and applied it to examine the level of fidelity of implementation 
of a hybrid instructional model for an upper division computer engineering course. The framework includes three components of intended curriculum, implemented curriculum, and learned curriculum. We've identified the driving forces behind the intended curriculum, including needs of society and workforce development, rapid advancement of content topics, and values and beliefs of the subject area. We recognized factors that influence the implemented curriculum, which encompass instructors' pedagogical and content knowledge as well as their perceived student needs. Factors such as limited availability of the instructor and intellectual openness of students' can be constraints for the curriculum implementation. The current study primarily compares the outcomes between the two different types of instructional methods.

\section{Course Curriculum Development}

The curriculum for the computer engineering course was previously designed. The course curriculum provides an introduction to fundamental concepts in the field of data communications and computer networks ${ }^{[3]}$. Survey questions designed and responses collected for this study were based on an upper division undergraduate computer engineering course, CEE 425 Data Communications and Computer Networking, at University of Wisconsin-Stout. The course was taught in Fall 2012 semester using hybrid method and in Fall 2013 semester using traditional inclassroom method. The course provides an introduction to fundamental concepts in the design and implementation of data communication networks, their protocols, and the associated applications. The objectives of this course aim to enable engineering students in understanding, evaluating, designing and implementation of computer networks. Derived from the course syllabus, the course content covers a list of topics shown in Table I. The course setup for the hybrid method was previously described ${ }^{[6]}$. In the traditional in-classroom method, the class is an instructor led which held regularly twice a week.

Table I. List of the Course Topics ${ }^{[4]}$

\begin{tabular}{|c|c|}
\hline $\begin{array}{l}\text { 1. Introduction to Internet } \\
\circ \text { Circuit Switching vs. Packet Switching } \\
\circ \text { Basic Network Performance Metrics } \\
\circ \text { OSI/Internet Model } \\
\end{array}$ & $\begin{array}{l}\text { 6. Wireless and Mobile Network } \\
\circ \text { Wireless Protocol Overview } \\
\circ \text { CDMA } \\
\circ \text { CSMA/CA } \\
\end{array}$ \\
\hline $\begin{array}{l}\text { 2. Application Layer } \\
\circ \text { HTTP, FTP, DNS, SMTP } \\
\circ \text { Socket Programming Concepts } \\
\end{array}$ & $\begin{array}{l}\text { 7. Multimedia Networking } \\
\circ \text { SIP } \\
\circ \text { Quality of Service (QoS) } \\
\end{array}$ \\
\hline $\begin{array}{ll}\text { 3. } & \text { Transport Layer } \\
\circ & \text { Reliable Data Transfer } \\
\circ & \text { TCP, UDP } \\
\circ & \text { Flow/Congestion Control } \\
\end{array}$ & $\begin{array}{l}\text { 8. Network Security } \\
\circ \text { Principles of Network Security } \\
\circ \text { IPSec, SSL, VPN }\end{array}$ \\
\hline $\begin{array}{ll}\text { 4. Network Layer } \\
\circ \text { Routing Principles (LS, DV) } \\
\circ \text { Routing Algorithms (RIP, OSPF, BGP) } \\
\circ \text { Internet Protocol (IP) } \\
\end{array}$ & $\begin{array}{ll}\text { 9. } & \text { Network Management } \\
\circ & \text { SNMP Operations } \\
\circ & \text { Management Information Base } \\
\circ & \text { Network Management Systems } \\
\end{array}$ \\
\hline $\begin{array}{ll}\text { 5. Data Link Layer } \\
\circ \text { Error Detection/Correction } \\
\circ \text { Multiple Access Protocols } \\
\circ \text { MAC, Ethernet, LAN Switches, VLAN } \\
\end{array}$ & \\
\hline
\end{tabular}




\section{Course Outcomes Comparison}

The outcomes of the study are presented herein by comparing the two teaching methods based on the survey data collected and final grade results. Survey questions were identical and same method of collecting the survey data was administrated between the two classes. Table II describes the first set of the survey questions. Comparisons of the results are shown in Figure $\mathbf{1 .}$ While both classes received good overall ratings (i.e. $>3$ or better, except for T3 question) in all survey questions, the results indicated that traditional in-classroom method appears to be more favored by the computer engineering students. Collected survey data were insufficient to further analyze the gap illustrated in T3 question.

Table II. Survey Questions shown in Figure 1

\begin{tabular}{|l|l|}
\hline Label & Survey Question \\
\hline T1 & $\begin{array}{l}\text { The instructor makes students aware of the content and skills to be } \\
\text { learned in the course. }\end{array}$ \\
\hline T2 & The instructor understands students' learning difficulties in this class. \\
\hline T3 & $\begin{array}{l}\text { The instructor uses various techniques to teach new and difficult } \\
\text { concepts. }\end{array}$ \\
\hline T4 & $\begin{array}{l}\text { The instructor provides adequate instructions and encourages } \\
\text { students to explore their own ways of learning }\end{array}$ \\
\hline T5 & The instructor makes the course content interesting to learn. \\
\hline T6 & The instructor demonstrates willingness to help students' learning. \\
\hline
\end{tabular}

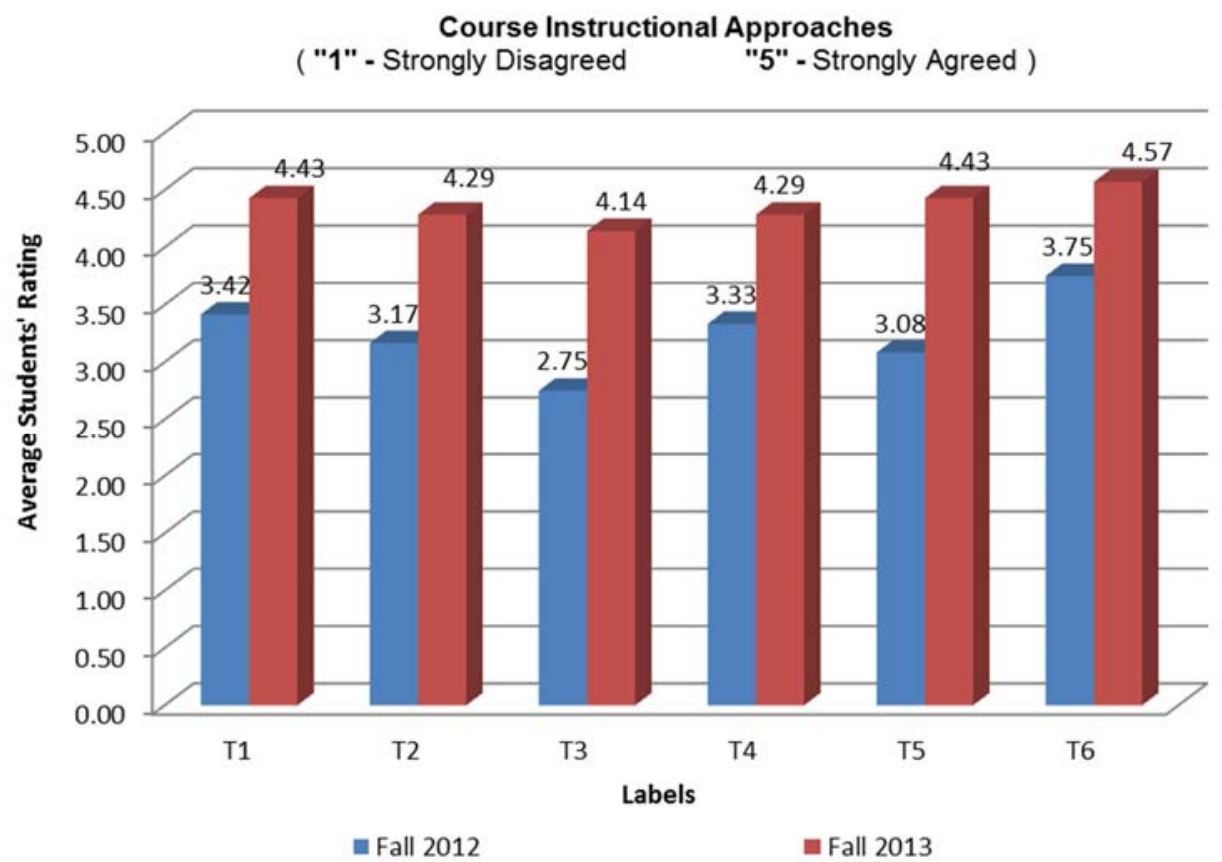

Figure 1. Students’ Rating of the Course Instructional Approaches 
Table III describes the second set of survey questions. Comparisons of the results are shown in Figure 2. In the team project evaluation, both classes indicated that team project did enhance their learning experiences in the field of data communications and computer networks. The ratings from both classes are mostly correlated to each other (i.e. $<0.5$ between the two values, except for L3 question). The correlation reflects that students were able to grasp theoretical concepts learned from working on the team project, independent of how each instructional method took place. The large gap in L3 question could be related to students not fully grasp theoretical concepts before starting to work on the team project in the hybrid instructional method. More analysis may be required to provide more insights.

Table III. Survey Questions shown in Figure 2

\begin{tabular}{|l|l|}
\hline Label & Survey Question \\
\hline L1 & The team project helps me improve my team work skills. \\
\hline L2 & The team project helps me improve my communication skills. \\
\hline L3 & $\begin{array}{l}\text { The team project helps me relate what we've learned in the } \\
\text { classroom to the real world. }\end{array}$ \\
\hline L4 & $\begin{array}{l}\text { The team project strengthened my conceptual understanding of } \\
\text { the course content. }\end{array}$ \\
\hline L5 & The team project helps me improve my problem solving skills. \\
\hline L6 & The team project helps me appreciate group learning. \\
\hline
\end{tabular}

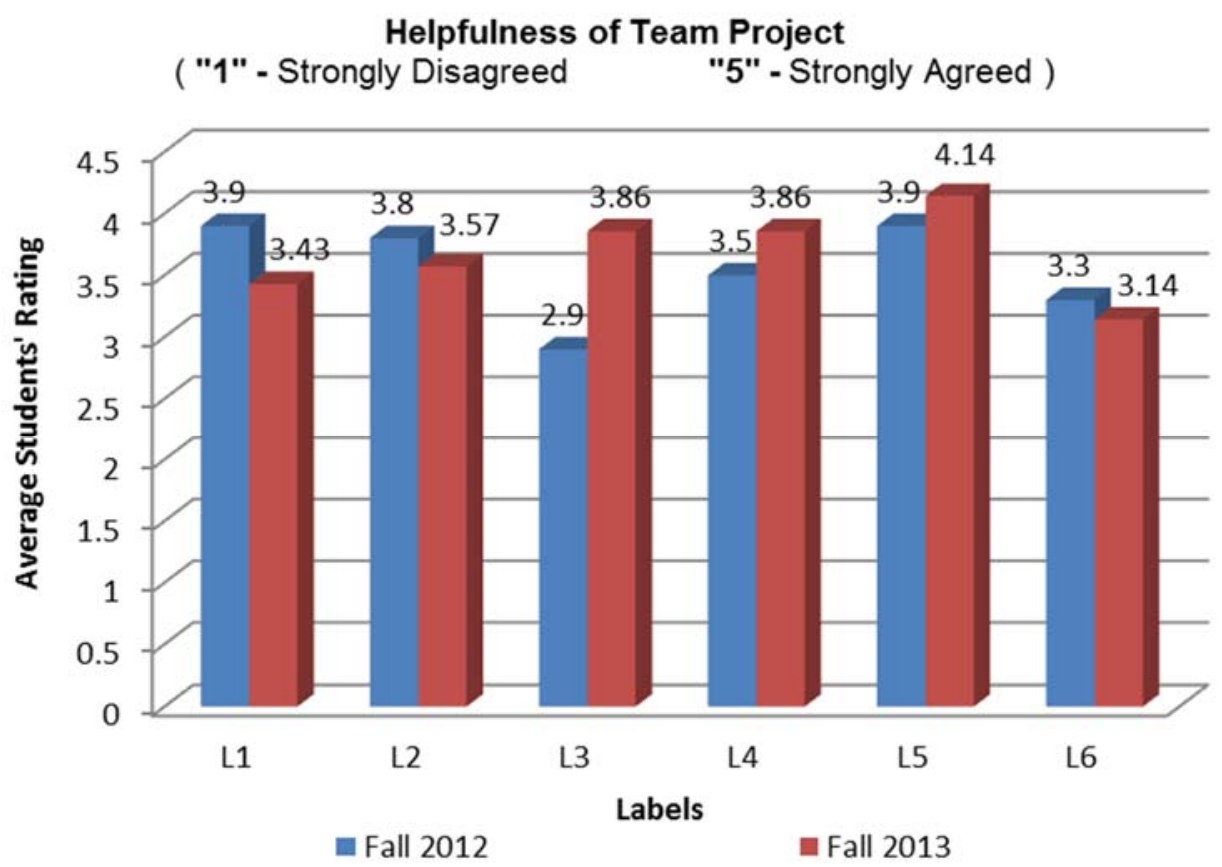

Figure 2. Students' Rating of the Team Project 
Finally, Figure 3 compares the final letter grade distribution between the two classes. It needs to be pointed out that the failing grade in Fall 2012 was an outlier in the data set since it was assigned to a special case, where a particular student decided not to show up to the class after few weeks into the semester. The student also did not drop the class either. In general, students from both classes performed reasonably well (i.e. $B$ or better). This also illustrates that students are able to perform reasonably well independent of the two instructional approaches.

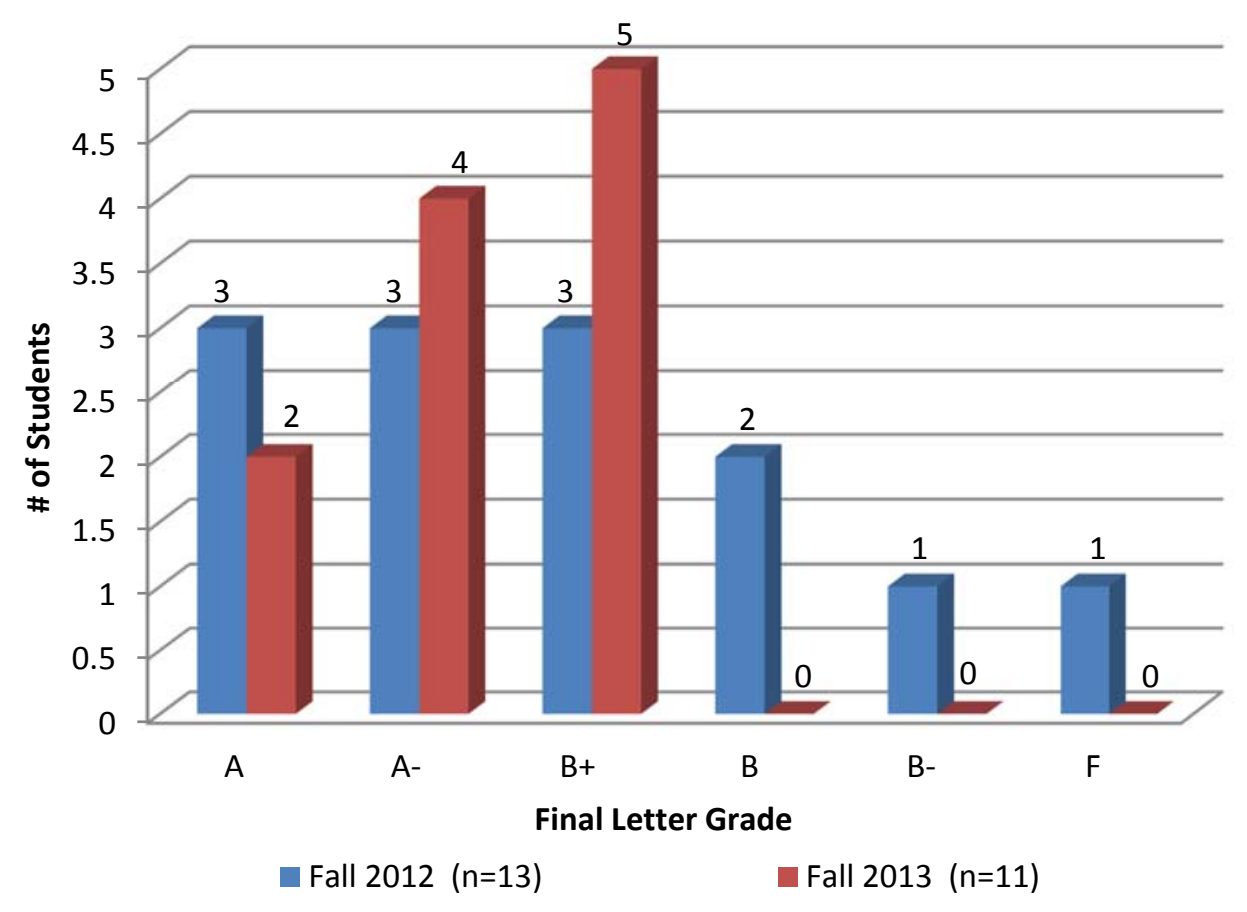

Figure 3. Comparison of Final Letter Grade Distribution

\section{Conclusion}

This paper compares hybrid and traditional in-classroom instructional methods based on a computer engineering course taught at UW-Stout. The results from survey questions were compared in order to assess the effectiveness of the designed course curriculum and to improve the development of the course content. While the design of the hybrid instructional method does fulfill similar requirements to the traditional in-classroom settings, the group of the students being surveyed still prefers traditional instructional method over the hybrid instructional method. Nevertheless, the study showed that students from both classes performed reasonably well. While we strongly believe that blending learning of a hybrid instructional model for computer engineering courses is a natural way to merge the implemented and learned curriculum into the intended curriculum, we find that if the learning activity is not carefully planned, students may not be as interested in an engineering course delivered using the hybrid instructional method. The transition from traditional in-classroom instructional method to hybrid or completely online method needs to be carefully planned in the future. 


\section{Bibliography}

[1]. Li, Chi-Sing, and Beverly Irby. "An Overview of Online Education: Attractiveness, Benefits, Challenges, Concerns and Recommendations." College Student Journal 42.2 (2008): 449-458.

[2]. Jia-Ling Lin, and Andy S. Peng, "A Framework to Examine Fidelity of Implementation of A Hybrid Instructional Model in Computer Engineering Courses”, the 43th ASEE/IEEE Frontiers in Engineering Education (FIE) Conference, 2013.

[3]. Andy S. Peng, Jia-Ling Lin, Robert Nelson, Cheng Liu, Ahmet Turkmen, Wei Shi, "Design and Development of a Hybrid Instructional Model for a Computer Engineering Course”, ASEE North Midwest Section Conference, Fargo, ND, 2013.

[4]. Kurose, James F., and Keith W. Ross. Computer Networking: A Top-Down Approach (6 ${ }^{\text {th }}$ Edition). Pearson Education, 2012. 